\title{
Gene-Environment Interaction in Parkinson's Disease: Coffee, ADORA2A, and CYP1A2
}

\author{
Yu-Hsuan Chuang ${ }^{a}$ Christina M. Lill ${ }^{f}$ Pei-Chen Lee ${ }^{h}$ Johnni Hansen ${ }^{i}$ \\ Christina F. Lassen ${ }^{i}$ Lars Bertram $^{g, j} \quad$ Naomi Greene ${ }^{a}$ \\ Janet S. Sinsheimer ${ }^{b, d}$ Beate Ritz ${ }^{a, c, e}$
}

${ }^{a}$ Department of Epidemiology, ${ }^{b}$ Department of Biostatistics, ${ }^{c}$ Department of Environmental Health Sciences, Fielding School of Public Health, University of California, Los Angeles (UCLA), dDepartment of Human Genetics and Biomathematics, and ${ }^{\mathrm{e}}$ Department of Neurology, David Geffen School of Medicine at UCLA, Los Angeles, CA, USA; ${ }^{\mathrm{f}}$ Genetic and Molecular Epidemiology Group, Institute of Neurogenetics, and IInterdisciplinary Platform for Genome Analytics (LIGA), Institutes of Neurogenetics and Integrative and Experimental Genomics, University

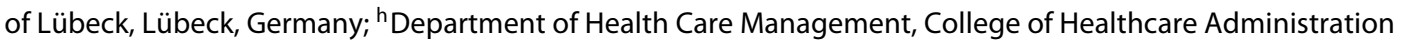
and Management, National Taipei University of Nursing Health Sciences, Taipei, Taiwan; 'Danish Cancer Society Research Center, Danish Cancer Society, Copenhagen, Denmark; ${ }^{j}$ School of Public Health, Faculty of Medicine, The Imperial College of Science, Technology, and Medicine, London, UK

\section{Keywords}

Parkinson's disease - Caffeine - Adenosine A2A receptor $(A D O R A 2 A) \cdot$ Cytochrome P450 1A2 (CYP1A2) · Meta-analysis

\footnotetext{
Abstract

Background and Purpose: Drinking caffeinated coffee has been reported to provide protection against Parkinson's disease (PD). Caffeine is an adenosine A2A receptor (encoded by the gene $A D O R A 2 A$ ) antagonist that increases dopaminergic neurotransmission and Cytochrome P450 1A2 (gene: CYP1A2) metabolizes caffeine; thus, gene polymorphisms in ADORA2A and CYP1A2 may influence the effect coffee consumption has on PD risk. Methods: In a population-based case-control study (PASIDA) in Denmark (1,556 PD patients and 1,606 birth year- and gender-matched controls), we assessed interactions between lifetime coffee consumption
}

and 3 polymorphisms in ADORA2A and CYP1A2 for all subjects, and incident and prevalent PD cases separately using logistic regression models. We also conducted a meta-analysis combining our results with those from previous studies. Results: We estimated statistically significant interactions for ADORA2A rs5760423 and heavy vs. light coffee consumption in incident (OR interaction $=0.66$ [95\% Cl 0.46-0.94], $p=$ 0.02 ) but not prevalent PD. We did not observe interactions for CYP1A2 rs762551 and rs2472304 in incident or prevalent PD. In meta-analyses, PD associations with daily coffee consumption were strongest among carriers of variant alleles in both ADORA2A and CYP1A2. Conclusion: We corroborated results from a previous report that described interactions between ADORA2A and CYP1A2 polymorphisms and coffee consumption. Our results also suggest that survivor bias may affect results of studies that enroll prevalent PD cases.

(C) 2017 S. Karger AG, Base

\section{KARGER}

E-Mail karger@karger.com www.karger.com/ned
(C) 2017 S. Karger AG, Basel

$0251-5350 / 17 / 0474-0192 \$ 39.50 / 0$
Beate Ritz, MD, PhD

Department of Epidemiology, Fielding School of Public Health University of California at Los Angeles, 650 Charles E. Young Drive Los Angeles, CA 90095-1772 (USA)

E-Mail britz@ucla.edu 


\section{Introduction}

More than $90 \%$ of Parkinson's disease (PD) is considered to be "idiopathic" - with genetic and environmental factors increasing risk of disease. A protective effect of coffee on PD has been postulated, since many epidemiologic studies reported lower consumption of caffeinated coffee among PD patients $[1,2]$. "Ever" vs. "never" drinkers have a $30 \%$ lower risk of PD and 3 additional cups of coffee per day lowered PD risk on average by $25-32 \%$ [3]. Caffeinated coffee is a very popular beverage in Northern European countries, especially Denmark, and we recently reported a $55 \%$ lower risk of PD among moderate coffee drinkers in Denmark [4]. A landmark early prospective cohort study not only reported an inverse association for coffee but also for caffeine from non-coffee sources, suggesting it might be the protective agent [5]. This association was replicated in many prospective cohort and case-control studies and further strengthened by observations of exposure-response trends [3, 6, 7]. Animal studies lent additional support to the idea that caffeine and its metabolites are neuro-protective [8]. Yet, the biological mechanisms underlying neuroprotection derived from caffeine are yet to be established. Importantly, epidemiologic data - even from prospective studies - do not preclude reverse causality since those who later develop PD may stop drinking caffeinated coffee due to sleep disorders, anxiety, gastro-intestinal problems or simply a loss of smell that could make coffee drinking less enjoyable in the very long pre-motor stages of PD. Evidence for interactions between caffeinated coffee and genes that metabolize caffeine or encode brain receptors targeted by caffeine, could help strengthen arguments that caffeine indeed plays a biological role in reducing PD risk.

Recently, a large consortium (PEGASUS) combined data from 1,325 PD cases and 1,735 controls and reported that PD risk was influenced by interactions between the single-nucleotide polymorphisms (SNP) rs5751876 and rs3032740 in ADORA2A, which encodes the adenosine A2A receptor in dopamine neurons, and caffeinated coffee consumption [9]; however, two much smaller studies did not find evidence for such interaction $[10,11]$. In addition, the PEGASUS study also observed stronger coffee-PD associations among carriers of the CC genotype of rs762551 in CYP1A2 compared with CA or AA carriers [9], a gene that encodes the cytochrome P450, family 1 , subfamily A, polypeptide 2 , the main caffeine-metabolizing enzyme.

Relying on data from a large population-based casecontrol study of PD (Parkinson's Disease in Denmark
[PASIDA]), here we re-examine interactions of coffee consumption with ADORA2A and CYP1A2 polymorphisms and also assess whether reliance on prevalent versus incident PD cases influences results, a distinction that may have caused previous study results to disagree [12].

\section{Method}

The PASIDA study was approved by the Institutional Review Boards of the Danish Data Protection Agency and the Ethics Committee of Copenhagen. Informed consent was obtained from all study participants.

Study Population

The PASIDA study enrolled idiopathic PD patients (ICD-8 342 and ICD-10 G20) treated at 10 neurological treatment centers and identified from the Danish National Hospital Register between 1996 and mid-2009 with subsequent validation of their diagnoses by medical record review. Population controls, free of PD when matched cases were diagnosed, were selected from the Danish Central Population Registry (individually matched on year of birth and gender). Detailed recruitment information was published previously [4]. Of 3,700 recruited subjects, 1,575 (87\%) PD cases and 1,607 (85\%) controls provided DNA samples (saliva) for genotyping. We further excluded subjects who were diagnosed with dementia prior to interview, leaving $1,556 \mathrm{PD}$ cases and 1,606 controls for analyses.

\section{Exposure Assessment and Variable Definition}

Standardized telephone interviews were conducted between 2008 and 2010 to obtain participants' lifetime caffeinated coffee consumption history (drip- and instant-coffee) and information on other lifestyle factors. Due to the high prevalence $(>90 \%)$ of caffeinated coffee drinking in Denmark, but little tea and caffeinated soda consumption during the study period, we omitted the latter caffeine sources. We collected lifetime amount and duration of caffeinated coffee-drinking, asking participants to report start and stop ages and the average number of cups they consumed per day. We consider an "ever" coffee drinker someone who consumed at least one cup $(6 \mathrm{oz})$ of coffee per week for a year. To obtain the amount of caffeine intake, we converted coffee cups per day into daily caffeine consumption (mg) using the US Department of Agriculture criteria [13]. Only consumption before the index date contributed to our exposure measures, that is, the date of first motor symptom recorded on the medical record, or the date of PD diagnosis for both cases and their matched controls.

\section{Genotyping}

DNA was extracted from saliva using standard protocols. Samples were genotyped on the QuantStudio ${ }^{\mathrm{TM}} 12 \mathrm{~K}$ Flex Real-Time PCR System using multiplex Taqman allelic discrimination assays (Applied Biosystems) according to the manufacturer's protocol. Each 384-well plate included 5\% HapMap CEU samples genotyped in duplicates across plates to assess genotyping accuracy. To control for genotyping quality, we excluded samples with genotyping efficiency less than $80 \%$ and SNPs with low genotyping efficiency $(<95 \%)$ and accuracy $(<99.5 \%)$; all 3 SNPs (rs5760423, rs762551, and rs2472304) in this study met these criteria. 
Statistical Analysis

We tested for deviation from the Hardy-Weinberg equilibrium among controls using Pearson's chi-square test (all $p \geq 0.05$ ). We broke the matched pairs and conducted unconditional logistic regression analyses adjusting for gender, birth year, and onset/index age to estimate marginal associations between caffeinated coffee consumption and PD status as well as between the three ADORA2A or CYP1A2 polymorphisms and PD status (additive genetic model), respectively. We broke the matched sets to avoid loss of entire pairs with only one subject when conducting stratified analyses and to increase efficiency since many pairs shared the same matching variable values [4]. However, we compared the overall results from conditional with the results from unconditional logistic regression adjusted for all of the matching variables and found them to be identical. Matching variables (i.e., year of birth, gender, and onset/index age), potential confounders (i.e., any kind of tobacco smoking) and strong predictors of PD (i.e., family history) were included in all models. We treated coffee intake as a binary variable with light vs. heavy consumption (defined as 0 to $\leq$ median vs. >median cup-years [14]) and also as a continuous variable (number of cups per day). We further created categories of caffeine intake in $\mathrm{mg}$ per day and years of coffee consumed using category definitions from our previous paper [15]. The Wald test for trend was applied to categorized coffee variables testing for a linear relationship with PD. Information about ethnic diversity was not available, but based on demographics of the Danish population provided by Denmark, we are confident that the large majority were non-Hispanic Whites [16].

We used multiplicative terms in logistic regression adjusted for confounders to assess whether the ADORA2A or CYP1A2 polymorphisms modify caffeine-PD associations, and the likelihood ratio chi-square tests was used to evaluate statistical significance. We also restricted all analyses to incident PD patients and their matched controls, that is, those diagnosed close to their date of interview during 2006-2009, to assess whether survival or recall bias may have influenced results with prevalent patients. Analyses were conducted using SAS version 9.4 (SAS Institute, Inc., Cary, NC, USA).

Lastly, we conducted meta-analyses to aggregate results from PASIDA (incident cases only) and non-Hispanic Whites from PEGASUS [9], based on the type of PD case (i.e., incident), control selection (i.e., population-based controls), and ethnicity (i.e., nonHispanic Whites) using the metagen package in the $\mathrm{R}$ environment, which allows to fit fixed-effects and random-effects models [17]. In the meta-analysis, results of ADORA2A rs5760423 in PASIDA were equated with rs5751876 in PEGASUS because they are in high linkage disequilibrium (LD) [18]; also, we combined our coffee category of "heavy use" with "ever" consumption in PEGASUS as well as and "light" consumption in PASIDA with "never" in PEGASUS, since less than $10 \%$ of PASIDA participants reported having never consumed coffee.

\section{Results}

Our initial analysis included 3,162 Danish participants in the PASIDA study with high-quality genotyping data. The average age of PD onset or index age was 61 years for all participants (Table 1) and 64 years for incident PD patients and their matched controls only. Sixty percent of participants were male and, compared with population controls, PD cases were more likely to have a positive family history of PD and smoke less. Ninety-four percent of PD cases and $97 \%$ of controls were "ever" coffee consumers.

Heavy coffee drinking in PASIDA is associated with a $25 \%$ lower risk of PD (OR 0.75 [95\% CI 0.64-0.88]), and each additional cup of coffee consumed per day on average is associated with a $4 \%$ lower PD risk (OR 0.96 [95\% CI 0.93-0.99]); online suppl. Table S1; for all online suppl. material, see www.karger.com/doi/10.1159/000450855); inverse coffee-PD associations are estimated for both prevalent and incident PD. Of note, the per-cup measure of daily coffee consumption was not associated with $\mathrm{PD}$ among incident cases. OR estimates adjusted solely for birth year, gender, and onset/index age did not substantially differ from estimates further adjusted for "ever" smoking and PD family history. Finally, marginal associations of ADORA2A rs5760423 as well as of CYP1A2 rs762551 and rs2472304 (in LD with rs762551: $r^{2}=0.87$, $\left.\mathrm{D}^{\prime}=0.99\right)$ with PD status (incident and prevalent) were null (online suppl. Table S2).

Interaction analyses based on all subjects did not show statistically and significantly varying effects of caffeine across genotypes of ADORA2A or CYP1A2 polymorphisms, respectively (Table 2 ). However, there appeared to be a trend in coffee-PD effect estimates across ADORA2A rs5760423 genotypes: the OR for PD among heavy coffee drinkers, relative to light coffee drinkers, was 0.81 (95\% CI 0.62-1.05) for GG carriers compared with 0.68 (95\% CI $0.55-0.86)$ for GT and 0.54 (95\% CI $0.37-$ 0.78 ) for TT carriers (OR interaction $=0.85$ [95\% CI $0.68-$ $1.06], p$ for interaction $=0.14)$. Further adjustment for smoking and PD family history did not change results (data not shown). When we restricted our analyses to incident PD only, we observed a statistically significant interaction for the ADORA2A rs5760423 and heavy coffee drinking (OR interaction $=0.66$ [95\% CI 0.46-0.94], $p$ for interaction $=0.02)$ : the OR for drinking coffee in GG carriers was 1.10 (95\% CI 0.72-1.68), 0.63 (95\% CI $0.44-$ 0.92 ) for GT, and 0.58 (95\% CI 0.30-1.09) for TT carriers. When the duration of caffeine intake was removed from the caffeine measure, the interaction of ADORA2A polymorphism and coffee was not statistically significant ( $p$ for interaction $=0.28$ in cup/day for all cases, and $p=$ 0.55 for incident cases, respectively). There was no evidence for association measure modification for CYP1A2 rs762551 and rs2472304 $(p$ for interaction $=0.45$ and 


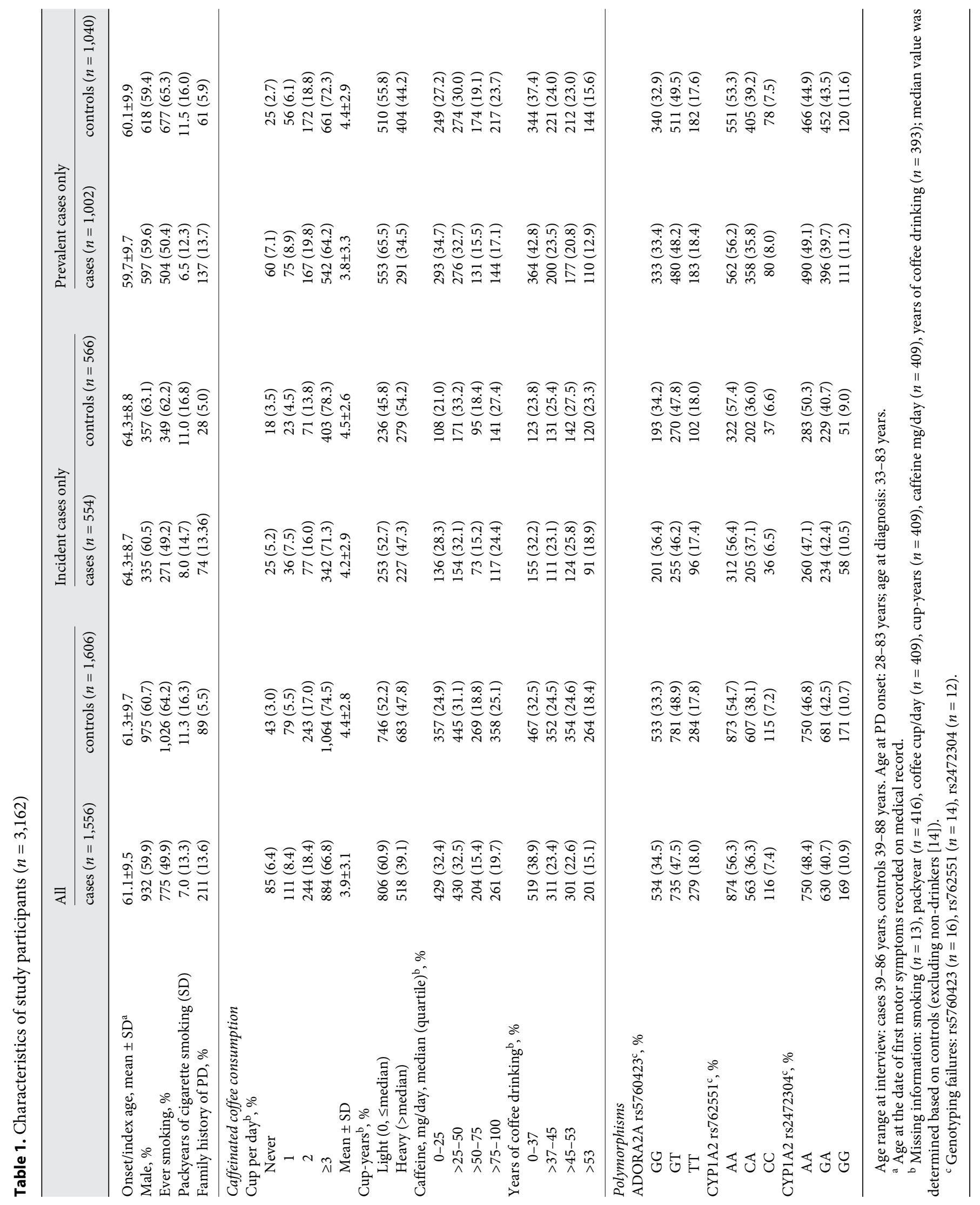




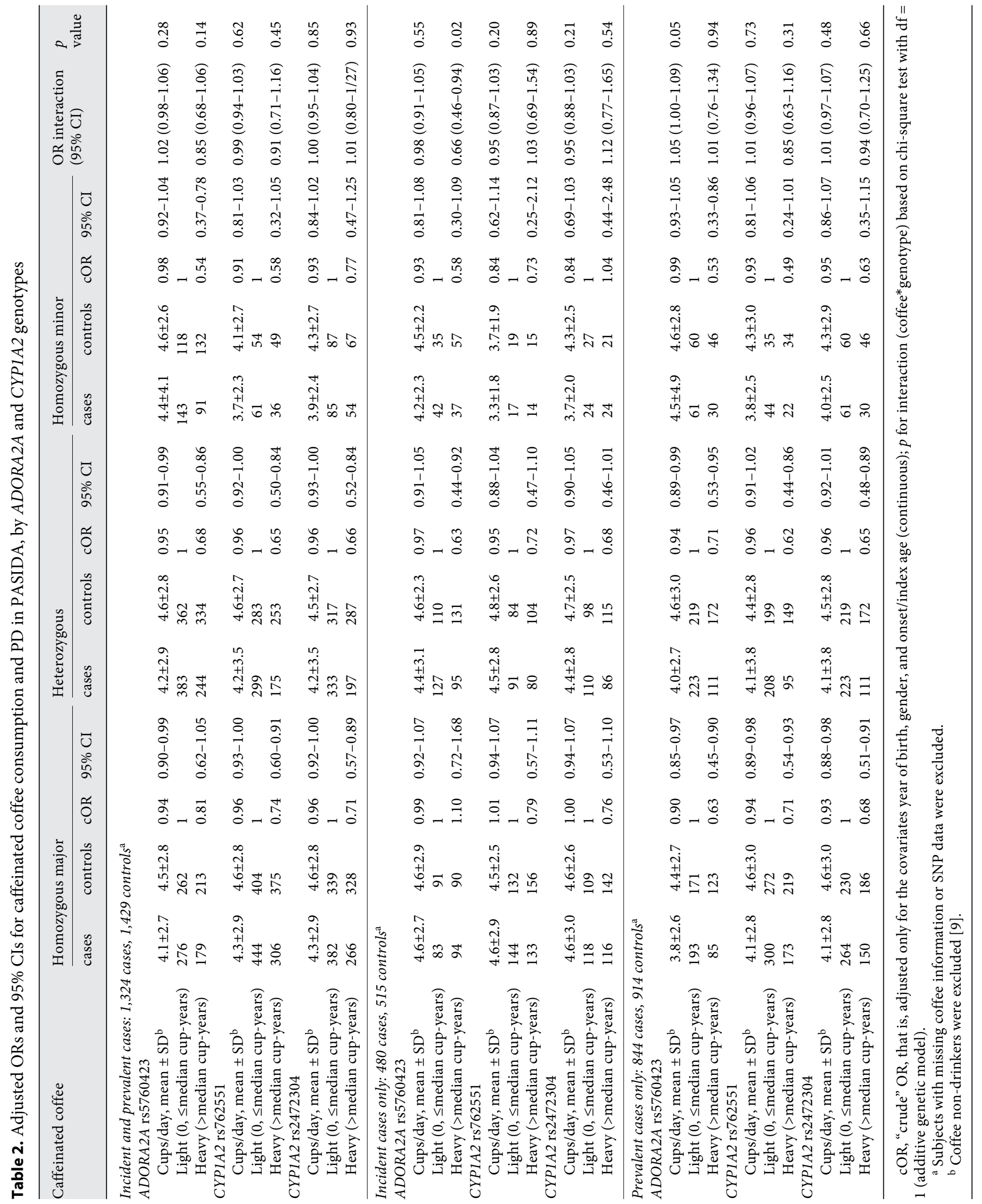


Table 3. Adjusted ORs and 95\% CIs for the association between caffeinated coffee consumption and PD in the PEGASUS and PASIDA studies, by $A D O R A 2 A$ and $C Y P 1 A 2$ genotypes: meta-analytic results using random-effect models

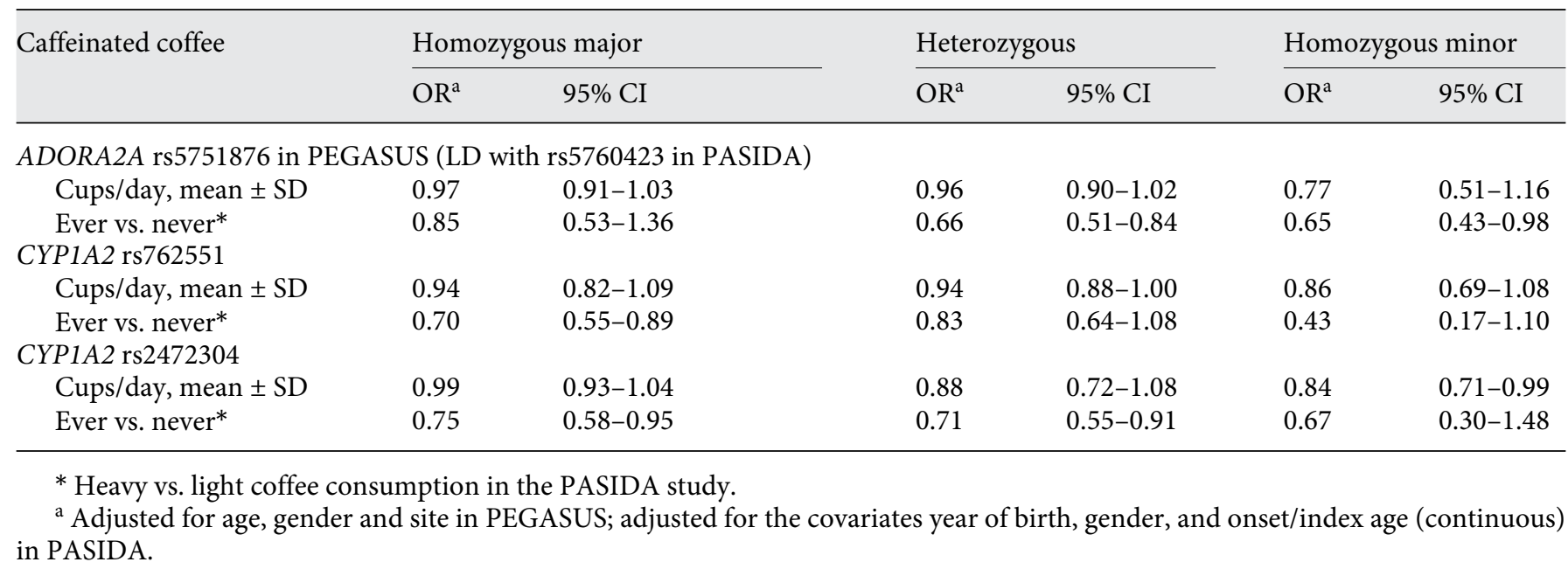

0.93, respectively). Similarly, restricting to incident cases only did not reveal statistically significant interactions for CYP1A2 SNPs. No interactions were found in prevalent case analyses. Results of interaction analyses using other coffee measures, daily intake of caffeine and years of coffee drinking are presented in online supplemental Table S3. We did not observe evidence for effect-measure modification with these measures and the SNPs we investigated.

Our meta-analytic results for coffee-PD associations did not differ much when we used random-effects versus fixed-effects models (Table 3; online suppl. Table S4). Based on random effects models, the ADORA2A gene polymorphisms and daily coffee consumption association for PD was strongest among rs5760423 TT carriers, OR 0.77 (95\% CI 0.51-1.16), compared with GT and GG carriers (OR 0.96 [95\% CI 0.90-1.02] and 0.97 [95\% CI 0.911.03], respectively). We saw similar patterns for CYP1A2 polymorphisms in both rs762551 and rs24702304, that is, the coffee-PD associations were strongest among homozygotes for the variant alleles (OR 0.86 [95\% CI 0.69-1.08] for rs762551 CC carriers and 0.84 [95\% CI 0.71-0.99] for rs24702304 GG carriers; Fig. 1).

\section{Discussion}

We conducted analyses of gene-environment interactions in a Danish case-control study of PD. Our study follows up on the results from a previous consortium study (PEGASUS) with an equally large sample size. When we include both prevalent and incident PD cases in our analysis, we found no evidence for interactions with $A D O R A 2 A / C Y P 1 A 2$ polymorphisms. However, when we restricted analyses to incident cases only, we observed interactions between the ADORA2A polymorphism and coffee drinking. This difference in results suggests that survival or recall bias may affect studies that rely on or include prevalent PD cases. Moreover, when we combined our results for incident cases with the PEGASUS incident cases of European ancestry in a meta-analytical approach, both $A D O R A 2 A$ and $C Y P 1 A 2$ polymorphisms modified coffee-PD associations, although the CYP1A2 interaction was solely due to the influence of the PEGASUS study.

Our PASIDA findings are mostly consistent with those published by the PEGASUS consortium, which previously reported ORs for PD risk of each additional cup of coffee consumed per day among coffee drinkers as 0.93 (95\% CI 0.84-1.03) and 0.92 (95\% CI 0.81-1.04) for CC or CT carriers of ADORA2A rs5751876, respectively, and 0.61 $(95 \%$ CI $0.46-0.81)$ for TT carriers $(p$ for interaction $=$ 0.01 ) in non-Hispanic Whites [9]. Yet, two smaller studies did not find statistically significant interactions for ADORA2A polymorphisms and coffee in PD $[10,11]$. One study was conducted in a mixed-race population that did not find the expected inverse main effect for coffee consumption on PD, possibly because sibling controls were used [10]. Sibling controls are likely too similar to cases in terms of coffee consumption, making it hard to 


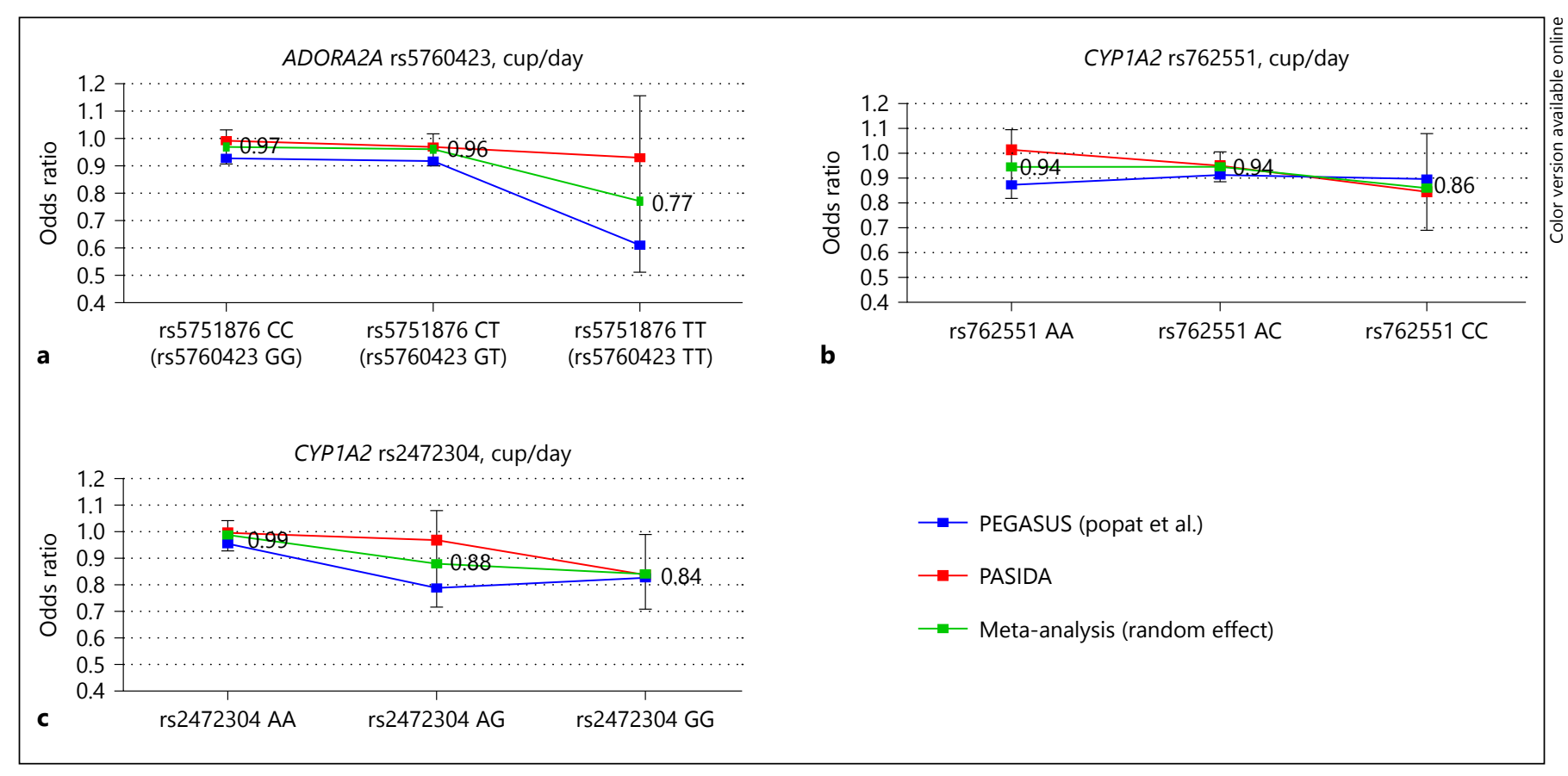

Fig. 1. a-c ORs for cups of coffee consumed per day and PD across ADORA2A/CYP1A2 gene polymorphisms.

estimate effects of coffee consumption on PD risk. The second null result was reported for an Asian population with a low average coffee consumption (2.9 in cases vs. 4.7 in controls [11] cup-years compared with 161.3 vs. 186.5 cup-years in PASIDA), such that the exposure levels and contrasts were likely insufficient.

Animal studies have shown that administration of caffeine or other adenosine A2A receptor antagonists before dosing the animal with the neurotoxin 1-methyl-4-phenyl-1,2,3,6-tetrahydropyridine reduces loss of dopamine and dopaminergic neurons, suggesting that caffeine reduces $\mathrm{PD}$ risk by deactivating the A2A receptors $[19,20]$. Also, the ADORA2A rs3032740 variant (in LD with rs5751876) has been shown to reduce protein expression [21], and thus may result in reduced A2A receptor function that together with further inhibition through coffee consumption may exert protective effects [9]. We would thus expect the inverse coffee-PD association to be the strongest in those with a TT genotype in ADORA2A rs5760423 (in LD with rs5751876). Adenosine A2A receptors have also become the latest target for non-dopaminergic therapies in PD based on their interaction with dopamine $\mathrm{D}_{2}$ receptors in striatopallidal neurons $[22,23]$.

Cytochrome P450 1A2 is the main caffeine-metabolizing enzyme that converts over $90 \%$ of caffeine in the liver to paraxanthine, and its activity depends on age, gen- der, smoking, and CYP1A2 polymorphisms [24-26]. Thus, we would expect neuroprotection due to caffeine to be stronger in slow metabolizers who carry the variant alleles we investigated. The PEGASUS consortium pooling incident case-control studies $(n=3,060)$, found evidence for coffee-CYP1A2 interactions with inverse PD associations for coffee drinking being strongest in CC genotype carriers at rs762551 and GG genotype carriers at rs2472304 [9]. The NeuroGenetic Research Consortium $(n=2,389)$ did not find interactions with CYP1A2 but included prevalent cases and some studies used spousal controls [12].

Previously, concerns were raised that confounding by population structure in PEGASUS produced spurious results $[12,27]$ since allele frequencies for CYP1A2 SNPs vary strongly across ethnicities and in PEGASUS the coffee-CYP1A2 interactions did not reach statistical significance in non-Hispanic Whites alone [9]. However, combining PEGASUS and PASIDA non-Hispanic Whites, our meta-analysis produced a decreasing trend across CYP1A2 rs762551 genotypes (green/middle line; Fig. 1b) and suggested an interaction with cups per day of consumption. Interestingly, "ever" (vs. "never") coffee consumption produced an inverted-U shape for the CYP1A2 rs762551 polymorphisms, but "ever (vs. "never") coffee consumption is a poor measure of average caffeine intake (Table 3 ). 
Smoking is positively associated with coffee drinking and negatively with PD risk, which we have previously interpreted as a consequence of pre-motor prodromal PD [3]. Moreover, a study reported that the metabolic activity did not differ between AC or CC and AA carriers at rs762551 in non-smokers suggesting that CYP1A2 genotypes may influence enzyme activity only in smokers [26]. In our study, adjustment for smoking did not change the interaction estimates for either of the CYP1A2 SNPs. Complicating the matter further, both caffeine and its CYP1A2 metabolite paraxanthine may non-selectively bind to adenosine receptor and act as a neuroprotector diminishing somewhat the potential importance of CYP1A2 enzyme activity [8]. The average Danish study participants drank as much as 4 cups per day over 40 years implying that levels of caffeine and its metabolites might be chronically higher than in other populations consuming less coffee possibly rendering the contributions of the metabolizing enzyme less important.

Our study has several strengths. We have a large sample size with a homogenous ancestry; we selected population controls from Danish registers, assessed confounding (including smoking) extensively, and were able to distinguish between incident and prevalent cases. High coffee consumption in Denmark allowed us to assess dose-response relationships for coffee and PD with great statistical power and since we collected detailed information on lifetime coffee consumption, we were able to define exposures in various ways.

Limitations to this study are that very few $(<10 \%)$ participants reported not drinking coffee such that CYP1A2 enzyme activity may not affect caffeine levels in the blood more than minimally making it hard to assess the influence of CYP1A2 polymorphisms. PD-prevalent cases tend to have more memory loss, and therefore lifetime coffee consumption could be misreported or reflect changes in drinking habits after diagnosis such as due to sleep problems common in PD patients. Also, recall might also be impaired in all cases and controls as the population was on average 68 years of age at the time of interview, which could cause non-differential exposure misclassification.

In conclusion, our study corroborates previous findings that interactions between ADORA2A rs5760423, CYP1A2 rs762551 and rs2472304 variants and coffee consumption affect PD risk. However, since our study only found interaction between ADORA2A rs5760423 and coffee for a measure of "total cup-years of coffee consumed" but not "average number of coffee cups per day," which is a measure used in the previous study, we cannot exclude the possibility that reverse causation contributed to these results. The lack of a cup-per-day association may, however, also be explained by the generally very high coffee consumption levels among Danes, that is, that few Danes consumed so little coffee that each additional cup would make a difference [9]. Therefore, additional data and studies are still needed in support of the hypothesis that a biological effect of caffeine protects against PD.

\section{Acknowledgement}

Y.C. was funded by the Burroughs Wellcome Fund InterSchool Training Program in Chronic Diseases in the past 12 months. The funding source had no role in the design, conduct, or analysis of the study. Research reported in this journal was supported by NIEHS of the National Institutes of Health under award number R01ES013717.

\section{Authors' Roles}

Y.C. and C.L. analyzed the data. Y.C. wrote the first draft. All co-authors contributed to study concept, design, and review and critique the manuscript.

\section{Financial Disclosures}

None.

\section{Disclosure Statement}

The authors have no conflicts of interest to declare.

\section{Funding}

This work was supported by National Institutes of Health grant R01ES013717.

References
1 Nefzger MD, Quadfasel FA, Karl VC: A retrospective study of smoking in Parkinson's disease. Am J Epidemiol 1968;88:149-158. bra BP, Vieregge P, Nischan P, Joerg J, Oertel WH, Schneider E, Ulm G: Diet and Parkinson's disease. I: a possible role for the past intake of specific foods and food groups. Results from a self-administered food-frequency questionnaire in a case-control study. Neurology 1996;47:636-643.
2 Hellenbrand W, Seidler A, Boeing H, Ro- 
3 Hernán MA, Takkouche B, Caamaño-Isorna F, Gestal-Otero JJ: A meta-analysis of coffee drinking, cigarette smoking, and the risk of Parkinson's disease. Ann Neurol 2002;52: 276-284.

4 Kenborg L, Lassen CF, Ritz B, Andersen KK, Christensen J, Schernhammer ES, Hansen J, Wermuth L, Rod NH, Olsen JH: Lifestyle, family history, and risk of idiopathic Parkinson disease: a large Danish case-control study. Am J Epidemiol 2015;181:808-816.

5 Ross GW, Abbott RD, Petrovitch H, Morens DM, Grandinetti A, Tung KH, Tanner CM, Masaki KH, Blanchette PL, Curb JD, Popper JS, White LR: Association of coffee and caffeine intake with the risk of Parkinson disease. JAMA 2000;283:2674-2679.

6 Saaksjarvi K, Knekt P, Rissanen H, Laaksonen MA, Reunanen A, Mannisto S: Prospective study of coffee consumption and risk of Parkinson's disease. Eur J Clin Nutr 2008;62: 908-915.

7 Qi H, Li S: Dose-response meta-analysis on coffee, tea and caffeine consumption with risk of Parkinson's disease. Geriatr Gerontol Int 2014;14:430-439.

$8 \mathrm{Xu} \mathrm{K,} \mathrm{Xu} \mathrm{YH,} \mathrm{Chen} \mathrm{JF,} \mathrm{Schwarzschild} \mathrm{MA:}$ Neuroprotection by caffeine: time course and role of its metabolites in the MPTP model of Parkinson's disease. Neuroscience 2010;167: 475-481.

9 Popat RA, Van Den Eeden SK, Tanner CM, Kamel F, Umbach DM, Marder K, Mayeux R, Ritz B, Ross GW, Petrovitch H, Topol B, McGuire V, Costello S, Manthripragada AD, Southwick A, Myers RM, Nelson LM: Coffee, ADORA2A, and CYP1A2: the caffeine connection in Parkinson's disease. Eur J Neurol 2011;18:756-765.

10 Facheris MF, Schneider NK, Lesnick TG, de Andrade M, Cunningham JM, Rocca WA, Maraganore DM: Coffee, caffeine-related genes, and Parkinson's disease: a case-control study. Mov Disord 2008;23:2033-2040.

11 Tan EK, Lu ZY, Fook-Chong SM, Tan E, Shen H, Chua E, Yih Y, Teo YY, Zhao Y: Exploring an interaction of adenosine $\mathrm{A} 2 \mathrm{~A}$ receptor variability with coffee and tea intake in Parkinson's disease. Am J Med Genet B Neuropsychiatr Genet 2006;141B:634-636.

12 Hill-Burns EM, Hamza TH, Zabetian CP, Factor SA, Payami H: An attempt to replicate interaction between coffee and CYP1A2 gene in connection to Parkinson's disease. Eur Neurol 2011;18:e107-e108; author reply e109.

13 Gebhardt SE, Thomas RG: Nitritive Value of Foods. Beltsville, US Department of Agriculture, Agricultural Research Service, Nutrient Data Laboratory, 2000, p 11.

14 Hamza TH, Chen H, Hill-Burns EM, Rhodes SL, Montimurro J, Kay DM, Tenesa A, Kusel VI, Sheehan P, Eaaswarkhanth M, Yearout D, Samii A, Roberts JW, Agarwal P, Bordelon Y, Park Y, Wang L, Gao J, Vance JM, Kendler KS, Bacanu SA, Scott WK, Ritz B, Nutt J, Factor SA, Zabetian CP, Payami H: Genomewide gene-environment study identifies glutamate receptor gene GRIN2A as a Parkinson's disease modifier gene via interaction with coffee. PLoS Genet 2011;7:e1002237.

15 Ahmed I, Lee PC, Lill CM, Searles Nielsen S, Artaud F, Gallagher LG, Loriot MA, Mulot C, Nacfer M, Liu T, Biernacka JM, Armasu S, Anderson K, Farin FM, Lassen CF, Hansen J, Olsen JH, Bertram L, Maraganore DM, Checkoway H, Ritz B, Elbaz A: Lack of replication of the GRIN2A-by-coffee interaction in Parkinson disease. PLoS Genet 2014; 10:e1004788.

16 Danmarks Statistik: Indvandrere i Danmark 2015. 2015. http://www.dst.dk/da/Statistik/ Publikationer/VisPub?cid=20703.

17 Friedrich T: Generalised Inference in the Random Effects Meta Regression Model. 2013. http://www2.uaem.mx/r-mirror/web/ packages/metagen/metagen.pdf.

18 Byrne EM, Johnson J, McRae AF, Nyholt DR, Medland SE, Gehrman PR, Heath AC, Madden PA, Montgomery GW, Chenevix-Trench G, Martin NG: A genome-wide association study of caffeine-related sleep disturbance: confir- mation of a role for a common variant in the adenosine receptor. Sleep 2012;35:967-975.

19 Chen JF, Xu K, Petzer JP, Staal R, Xu YH, Beilstein $\mathrm{M}$, Sonsalla PK, Castagnoli K, Castagnoli N Jr, Schwarzschild MA: Neuroprotection by caffeine and $\mathrm{A}(2 \mathrm{~A})$ adenosine receptor inactivation in a model of Parkinson's disease. J Neurosci 2001;21:RC143.

20 Pierri M, Vaudano E, Sager T, Englund U: KW-6002 protects from MPTP induced dopaminergic toxicity in the mouse. Neuropharmacology 2005;48:517-524.

21 Alsene K, Deckert J, Sand P, de Wit H: Association between $\mathrm{A} 2 \mathrm{~A}$ receptor gene polymorphisms and caffeine-induced anxiety. Neuropsychopharmacology 2003;28:1694-1702.

22 Ferre S, Quiroz C, Woods AS, Cunha R, Popoli P, Ciruela F, Lluis C, Franco R, Azdad K, Schiffmann SN: An update on adenosine A2Adopamine D2 receptor interactions: implications for the function of $\mathrm{G}$ protein-coupled receptors. Curr Pharm Des 2008;14:1468-1474.

23 Franco R, Ferre S, Agnati L, Torvinen M, Gines S, Hillion J, Casado V, Lledo P, Zoli M, Lluis C, Fuxe K: Evidence for adenosine/dopamine receptor interactions: indications for heteromerization. Neuropsychopharmacology 2000;23(4 suppl):S50-S59.

24 Gunes A, Dahl ML: Variation in CYP1A2 activity and its clinical implications: influence of environmental factors and genetic polymorphisms. Pharmacogenomics 2008;9:625-637.

25 Backman JT, Schroder MT, Neuvonen PJ: Effects of gender and moderate smoking on the pharmacokinetics and effects of the CYP1A2 substrate tizanidine. Eur J Clin Pharmacol 2008;64:17-24.

26 Sachse C, Brockmoller J, Bauer S, Roots I: Functional significance of a C->A polymorphism in intron 1 of the cytochrome $\mathrm{P} 450$ CYP1A2 gene tested with caffeine. Br J Clin Pharmacol 1999;47:445-449.

27 Mellick GD, Ross OA: Caffeine and Parkinson's disease: are we getting our fix on riskmodifying gene-environment interactions? Eur J Neurol 2011;18:671-672. 